\title{
A Fundamental Relationship of Polynomials and Its Proof
}

\author{
Serdar Beji \\ Faculty of Naval Architecture and Ocean Engineering, Istanbul Technical University, Istanbul, Turkey \\ Email: sbeji@itu.edu.tr
}

How to cite this paper: Beji, S. (2018) A Fundamental Relationship of Polynomials and Its Proof. Advances in Pure Mathematics, 8, 559-563.

https://doi.org/10.4236/apm.2018.86033

Received: May 21, 2018

Accepted: June 23, 2018

Published: June 26, 2018

Copyright (C) 2018 by author and Scientific Research Publishing Inc. This work is licensed under the Creative Commons Attribution International License (CC BY 4.0).

http://creativecommons.org/licenses/by/4.0/

\begin{abstract}
A fundamental algebraic relationship for a general polynomial of degree $n$ is given and proven by mathematical induction. The stated relationship is based on the well-known property of polynomials that the $n^{\text {th }}$-differences of the subsequent values of an $n^{\text {th }}$-order polynomial are constant.
\end{abstract}

\section{Keywords}

Polynomials of Degree $n, n^{\text {th }}$-Order Finite-Differences, Recurrence

Relationship for Polynomials

\section{Introduction}

The "Fundamental Theorem of Algebra" states that a polynomial of degree $n$ has $n$ roots. Its first assertion in a different form is attributed to Peter Rothe in 1606 and later Albert Girard in 1629. Euler gave a clear statement of the theorem in a letter to Gauss in 1742 and at different times Gauss gave four different proofs (see [1], p. 292-306).

A nearly as important property of a polynomial is the constancy of the $n^{\text {th }}$-differences of its subsequent values. To clarify this point let us begin with some demonstrations. While it is customary to use polynomials with real coefficients, here a second-order polynomial with complex coefficients is considered first,

$$
P_{2}(x)=(1+i) x^{2}-3 i x+2
$$

where $i=\sqrt{-1}$ is the imaginary unit. Taking a real starting point $x_{0}=-2$ and a real step value $s=1$ the following Table 1 of differences can be established for the subsequent values of the polynomial.

The first differences are computed by taking the differences of the subsequent values of the polynomial as in $P_{2}(-2)-P_{2}(-1)=(6+10 i)-(3+4 i)=3+6 i$. 
Table 1. Second-order differences for a sample second-order polynomial.

\begin{tabular}{|c|c|c|c|c|c|}
\hline$m$ & 0 & 1 & 2 & 3 & 4 \\
\hline$x_{0}+m s$ & -2 & -1 & 0 & 1 & 2 \\
\hline$P_{2}\left(x_{0}+m s\right)$ & $6+10 i$ & $3+4 i$ & $2+0 i$ & $3-2 i$ & $6-2 i$ \\
\hline
\end{tabular}
First differences
Second differences

The second differences are obtained similarly using the first difference values: $(3+6 i)-(1+4 i)=2+2 i$.

Expressing the first differences in terms of polynomial values $P_{2}(-2)-P_{2}(-1)=3+6 i$ and $P_{2}(-1)-P_{2}(0)=1+4 i$, the first value of the second differences may be written as

$$
\begin{aligned}
& {\left[P_{2}(-2)-P_{2}(-1)\right]-\left[P_{2}(-1)-P_{2}(0)\right]} \\
& =P_{2}(-2)-2 P_{2}(-1)+P_{2}(0)=2+2 i
\end{aligned}
$$

which is a particular form, $n=2$, of the general theorem presented in Section 2. The constant value $2+2 i$ of the second-differences can be calculated from the general expression $(-1)^{n} n ! a_{0} s^{n}$ where $n$ is the degree of the polynomial and $a_{0}$ the coefficient of the $n^{\text {th }}$-order term. For this particular example $n=2$ and $a_{0}=1+i$ hence the constant becomes $(-1)^{2} 2 !(1+i) 1^{2}=2+2 i$ as found above.

Another example is now given for a third-order polynomial with real coefficients

$$
P_{3}(x)=2 x^{3}-x^{2}-3 x+5
$$

In this example a complex starting point $x_{0}=1-i$ and a complex step value $s=-3+2 i$ are used so that Table 2 of differences is constructed, where the constant value of the third-differences can be calculated from the general formula $(-1)^{n} n ! a_{0} s^{n}$ as $(-1)^{3} 3 ! 2(-3+2 i)^{3}=-108-552 i$.

A direct connection with the finite-difference approximation of derivatives of a polynomial is of course possible. Finite-difference approximation of the third-derivative of a third-order polynomial is given by

$$
P_{3}^{\prime \prime \prime}(x)=\frac{P_{3}\left(x_{0}+3 s\right)-3 P_{3}\left(x_{0}+2 s\right)+3 P_{3}\left(x_{0}+s\right)-P_{3}\left(x_{0}\right)}{s^{3}}
$$

where $s$ is the incremental step. If the third-order polynomial is defined as $P_{3}(x)=a_{0} x^{3}+a_{1} x^{2}+a_{2} x+a_{3}$ its third-derivative is $P_{3}^{\prime \prime \prime}(x)=6 a_{0}$. Now using this in (4) results in

$$
P_{3}\left(x_{0}\right)-3 P_{3}\left(x_{0}+s\right)+3 P_{3}\left(x_{0}+2 s\right)-P_{3}\left(x_{0}+3 s\right)=-6 a_{0} s^{3}
$$

which exactly corresponds to the tabulated constant of third-differences. A remarkable point is that while finite-difference approximations are typically formulated for real and relatively small incremental step sizes, for the general expression no such restrictions apply: the incremental step $s$ may be complex and arbitrarily large while the result is always exact. 
Table 2. Third-order differences for a sample third-order polynomial.

\begin{tabular}{|c|c|c|c|c|c|c|c|c|c|c|c|}
\hline$m$ & \multicolumn{2}{|c|}{0} & \multicolumn{2}{|c|}{1} & \multicolumn{2}{|l|}{2} & \multicolumn{2}{|l|}{3} & \multicolumn{2}{|l|}{4} & 5 \\
\hline$x+m s$ & \multicolumn{2}{|c|}{$1-i$} & \multicolumn{2}{|c|}{$-2+i$} & \multicolumn{2}{|c|}{$-5+3 i$} & \multicolumn{2}{|c|}{$-8+5 i$} & \multicolumn{2}{|c|}{$-11+7 i$} & $-14+9 i$ \\
\hline$P_{3}(x+m s)$ & \multicolumn{2}{|c|}{$-2+i$} & \multicolumn{2}{|c|}{$4+23 i$} & \multicolumn{2}{|c|}{$24+417 i$} & \multicolumn{2}{|c|}{$166+1735 i$} & $538+4$ & $529 i$ & $1248+9351 i$ \\
\hline \multicolumn{2}{|c|}{ First differences } & \multicolumn{2}{|c|}{$-6-22 i$} & \multicolumn{2}{|c|}{$-20-394 i$} & \multicolumn{2}{|c|}{$-142-1318 i$} & \multicolumn{2}{|c|}{$-372-2794 i$} & \multicolumn{2}{|c|}{$-710-4822 i$} \\
\hline \multicolumn{3}{|c|}{ Second differences } & \multicolumn{2}{|c|}{$-14-372 i$} & \multicolumn{2}{|c|}{$-122-924 i$} & \multicolumn{2}{|c|}{$-230-1476 i$} & \multicolumn{2}{|c|}{$-338-2028 i$} & \\
\hline \multicolumn{3}{|c|}{ Third differences } & & \multicolumn{2}{|c|}{$-108-552 i$} & \multicolumn{2}{|c|}{$-108-552 i$} & \multicolumn{2}{|c|}{$-108-552 i$} & & \\
\hline
\end{tabular}

Finally, a possible application of (5) or its general form for an $n^{\text {th }}$-order polynomial, is its use as a recurrence formula for evaluating a given polynomial at equal intervals once the polynomial is evaluated at $n$ distinct points. For instance for a third-order polynomial it is sufficient to know $P_{3}\left(x_{0}\right), P_{3}\left(x_{0}+s\right)$, and $P_{3}\left(x_{0}+2 s\right)$ to obtain $P_{3}\left(x_{0}+3 s\right)$ from (5). Then, by setting $x_{0}$ to $x_{0}+s$ in (5), $P_{3}\left(x_{0}+4 s\right)$ can be obtained from the same recurrence relationship and continuing in this manner gives $P_{3}\left(x_{0}+5 s\right), P_{3}\left(x_{0}+6 s\right)$, etc. with considerably less arithmetic operations compared to straightforward evaluation of polynomial.

\section{Main Theorem and Proof}

The main theorem which expresses the constancy of $n^{\text {th }}$-order differences for an $n^{\text {th }}$-order polynomial is stated first and then proven by the method of induction.

\section{Theorem 1}

For an $n^{\text {th }}$-order polynomial $P_{n}(x)=a_{0} x^{n}+a_{1} x^{n-1} \cdots+a_{n-1} x+a_{n}$ with $a_{0} \neq 0$ the following relationship holds

$$
\sum_{m=0}^{n}(-1)^{m}\left(\begin{array}{l}
n \\
m
\end{array}\right) P_{n}(x+m s)=(-1)^{n} n ! a_{0} s^{n}
$$

where $n \geq 1$ and $a_{j}$ 's, $x, s \in \mathbb{R}$ or $\mathbb{C}$.

Proof of Theorem 1. The base case: Setting $n=1$ in (6) results in

$$
\left(\begin{array}{l}
1 \\
0
\end{array}\right) P_{1}(x)-\left(\begin{array}{l}
1 \\
1
\end{array}\right) P_{1}(x+s)=(-1)^{1} 1 ! a_{0} s^{1}
$$

Substituting $P_{1}(x)=a_{0} x+a_{1}$ and $P_{1}(x+s)=a_{0}(x+s)+a_{1}$ gives

$$
\left(a_{0} x+a_{1}\right)-\left[a_{0}(x+s)+a_{1}\right]=-a_{0} s
$$

which is correct.

The inductive step: Assuming that the statement (6) holds true for any integer $n$ it is now proven that it also holds true for $(n+1)$ :

$$
\sum_{m=0}^{n+1}(-1)^{m}\left(\begin{array}{c}
n+1 \\
m
\end{array}\right) P_{n+1}(x+m s)=(-1)^{n+1}(n+1) ! a_{0} s^{n+1}
$$

$P_{n+1}(\bar{x})$ can be expressed in terms of $P_{n}(\bar{x})$ as

$$
P_{n+1}(\bar{x})=\bar{x} P_{n}(\bar{x})+a_{n+1}
$$


Letting $\bar{x}=x+m s$ in (10) and using it in (9) result in

$$
\begin{aligned}
& \sum_{m=0}^{n+1}(-1)^{m}\left(\begin{array}{c}
n+1 \\
m
\end{array}\right)\left[(x+m s) P_{n}(x+m s)+a_{n+1}\right] \\
& =(-1)^{n+1}(n+1) ! a_{0} s^{n+1}
\end{aligned}
$$

Since $\sum_{m=0}^{n+1}(-1)^{m}\left(\begin{array}{c}n+1 \\ m\end{array}\right)=0$ for any $n$ (odd or even) the summation proportional to the constant $a_{n+1}$ vanishes, reducing (11) to

$$
\begin{aligned}
& x \sum_{m=0}^{n+1}(-1)^{m}\left(\begin{array}{c}
n+1 \\
m
\end{array}\right) P_{n}(x+m s)+s \sum_{m=0}^{n+1}(-1)^{m} m\left(\begin{array}{c}
n+1 \\
m
\end{array}\right) P_{n}(x+m s) \\
& =(-1)^{n+1}(n+1) ! a_{0} s^{n+1}
\end{aligned}
$$

Making use of $\sum_{m=0}^{n+1}\left(\begin{array}{c}n+1 \\ m\end{array}\right)=\sum_{m=0}^{n}\left(\begin{array}{l}n \\ m\end{array}\right)+\sum_{m=1}^{n+1}\left(\begin{array}{c}n \\ m-1\end{array}\right)$ ([2], p. 882) in the first summation above results in

$$
\begin{aligned}
& x\left[\sum_{m=0}^{n}(-1)^{m}\left(\begin{array}{l}
n \\
m
\end{array}\right) P_{n}(x+m s)+\sum_{m=1}^{n+1}(-1)^{m}\left(\begin{array}{c}
n \\
m-1
\end{array}\right) P_{n}(x+m s)\right] \\
& +s \sum_{m=0}^{n+1}(-1)^{m} m\left(\begin{array}{c}
n+1 \\
m
\end{array}\right) P_{n}(x+m s)=(-1)^{n+1}(n+1) ! a_{0} s^{n+1}
\end{aligned}
$$

By re-defining the running index in the second summation (13) becomes

$$
\begin{aligned}
& x\left[\sum_{m=0}^{n}(-1)^{m}\left(\begin{array}{c}
n \\
m
\end{array}\right) P_{n}(x+m s)+\sum_{m=0}^{n}(-1)^{m+1}\left(\begin{array}{l}
n \\
m
\end{array}\right) P_{n}[x+(m+1) s]\right] \\
& +s \sum_{m=0}^{n+1}(-1)^{m} m\left(\begin{array}{c}
n+1 \\
m
\end{array}\right) P_{n}(x+m s)=(-1)^{n+1}(n+1) ! a_{0} s^{n+1}
\end{aligned}
$$

Since $x$ may be assigned to any value, substituting $x+s$ in place of $x$ in the base case (6) reveals that $\sum_{m=0}^{n}(-1)^{m}\left(\begin{array}{l}n \\ m\end{array}\right) P_{n}[x+(m+1) s]$ is too equal to the same quantity: $(-1)^{n} n ! a_{0} s^{n}$. Noting in the second summation in (14) that $(-1)^{m+1}=-(-1)^{m}$ renders the terms in square brackets zero. Thus, to complete the proof the remaining equality in the second line of (14) must be proven:

$$
s \sum_{m=0}^{n+1}(-1)^{m} m\left(\begin{array}{c}
n+1 \\
m
\end{array}\right) P_{n}(x+m s)=(-1)^{n+1}(n+1) ! a_{0} s^{n+1}
$$

For $m=0$ the first term of the summation in (15) is zero hence bringing no contribution. Therefore, we can start the summation from $m=1$ without any error. Then, the summation may be expressed as

$$
\begin{aligned}
& \sum_{m=1}^{n+1}(-1)^{m} m\left(\begin{array}{c}
n+1 \\
m
\end{array}\right) \\
& =\sum_{m=1}^{n+1}(-1)^{m} m \frac{(n+1) !}{(n+1-m) ! m !} \\
& =\sum_{m=1}^{n+1}(-1)^{m} \frac{(n+1) !}{(n+1-m) !(m-1) !}
\end{aligned}
$$




$$
\begin{aligned}
& =\sum_{m=1}^{n+1}(-1)^{m}(n+1) \frac{n !}{(n+1-m) !(m-1) !} \\
& =\sum_{p=0}^{n}(-1)^{p+1}(n+1) \frac{n !}{(n-p) ! p !} \\
& =(n+1) \sum_{p=0}^{n}(-1)^{p+1}\left(\begin{array}{l}
n \\
p
\end{array}\right)
\end{aligned}
$$

where an obvious change of running index $m=p+1$ has been implemented in the final stage. Employing the last expression obtained above after changing $p$ to $m$ for the summation of (15) results in

$$
s(n+1) \sum_{m=0}^{n}(-1)^{m+1}\left(\begin{array}{l}
n \\
m
\end{array}\right) P_{n}[x+(m+1) s]=(-1)^{n+1}(n+1) ! a_{0} s^{n+1}
$$

As indicated above, the main theorem may also be stated as

$$
\begin{gathered}
\sum_{m=0}^{n}(-1)^{m}\left(\begin{array}{l}
n \\
m
\end{array}\right) P_{n}[x+(m+1) s]=(-1)^{n} n ! a_{0} s^{n} \text {. Using this in (16) yields } \\
s(n+1)(-1)(-1)^{n} n ! a_{0} s^{n}=(-1)^{n+1}(n+1) ! a_{0} s^{n+1}
\end{gathered}
$$

which proves that the proposition holds true for $(n+1)$ as well.

\section{References}

[1] Smith, D.E. (1959) A Source Book in Mathematics. Dover Publications, New York.

[2] Abramowitz, M. and Stegun, I.A. (1972) Handbook of Mathematical Functions. Dover Publications, New York. 